\title{
THE NOTATION OF PENSION FUND PROBLEMS.
}

To the Editor of the Tourmal of the Institute of Actuaries.

SIR,-The different notations employed by Mr. King and Mr. Manly in their papers on Pension Funds are likely to be confusing to those who may find it desirable-as suggested in the reeent discussion - to read those papers together, and it would seem that the time has arrived when the Council of the Institute might profitably consider the question of settling and prescribing an official notation. As a preliminary, I venture to ask Mr. King to reconsider his symbols (for which Mr. Manly has no equivalent) for the various valuation multipliers or "factors." For all these functions, though representing very various benefits, Mr. King uses the one generic symbol F, which acts merely as a scaffolding on which to build up a number of subsidiary symbols serving to distinguish one function from another. This sacrifices-as it would seem, needlessly-the great advantages secured by the fundamental prineiple of the Institute notation, namely, that the principal symbol, which first strikes the eye, determines the nature of the function, while the small letters denote particular ages, terms of years, etc. I suggest that separate principal letters be used for withdrawal, death and pension benefits, and that a eapital letter denote benefits in respect of past contributions or salaries, and a small italic letter benefits in respect of future contributions or benefits. The particular letters are immaterial, but if Mr. King would substitute another symbol, say, $w_{x}^{h_{h}}$ for his $h_{x}$ (which is but rarely wantea and is not in fact used in any of his formulas), we should have the three 
consecutive letters $F, G$ and $H$ available for withdrawals, deaths and pensions respectively. That this would introduce a considerable simplification will be seen by the following comparisons :

Withdrawal BeNefits: IN REsPect of-

Past contributions . . . . . . $\mathbf{F}_{x}$ instead of ${ }^{p} \mathbf{F}_{x}^{w}$ Future $" \quad . \quad . \quad . \quad . \quad \cdot f_{x} \quad " \quad{ }^{\prime} \mathrm{F}_{x}^{w}$

Death Benefts: In Respect of-

Past contributions . . . . . . . $\quad \mathbf{G}_{x} \quad, \quad{ }^{p} \mathbf{F}_{x}^{d}$ Future " . . . . . $\quad g_{x} \quad, \quad{ }^{f} \mathbf{F}_{x}^{d}$

Past contributions with interest $. \quad \cdot{ }_{y} G_{x}^{i} \quad, \quad{ }_{y}^{p} \mathrm{~F}_{x}^{d i}$

Future contributions with interest . . $g_{x}^{i} \quad, \quad{ }^{f} \mathrm{~F}_{x}^{d i}$

Pension Benefits: In Respect of-

Past salaries-Unlimited Pensions . . $\mathbf{H}_{x} \quad,{ }^{p} \mathbf{F}_{x}^{r a}$ $" \quad, \quad$ Pensions with limitations . ${ }_{y} \mathrm{H}_{x} \quad, \quad{ }_{y}^{p} \mathrm{~F}_{x}^{r a}$

Future salaries-Unlimited Pensions $\quad . h_{x} \quad, \quad{ }^{{ }^{F}}{ }_{x}^{r a}$ $" \quad, \quad$ Pensions with limitations ${ }_{y} h_{x} \quad, \quad{ }_{y}^{f} \mathrm{~F}_{x}^{r a}$

The relatively few eases in which the benefit relates both to past and future contributions or salaries could conveniently be represented by a hollow capital letter.

That the conventional symbols $\mathrm{F}, f, \mathrm{G}, g, \mathrm{H}$, and $h$ would be readily learnt is conclusively proved by experience of the similar symbols in the Institute notation, and the suggested new notation would certainly be more easily written, printed, and read than that used by Mr. King.

It would trespass too much on your space to discuss in detail the symbols for commutation functions, but here also it would be desirable, if practicable, to introduee some simplification. In any case, much is gained by having a simple and easily-written notation for the factors which are actually used in a valuation.

I am, Sir, your obedient Servant,

6 February $1905 . \quad$ G. J. LIDSTONE. 